ACCEPTED MANUSCRIPT

\title{
Breakdown of the coherence effects and Fermi liquid behavior in $\mathrm{YbAl}_{3}$ nanoparticles
}

To cite this article before publication: Cristina Echevarria Bonet et al $2018 \mathrm{~J}$. Phys.: Condens. Matter in press https://doi.org/10.1088/1361$\underline{648 \mathrm{X} / \mathrm{aab0c7}}$

\section{Manuscript version: Accepted Manuscript}

Accepted Manuscript is "the version of the article accepted for publication including all changes made as a result of the peer review process, and which may also include the addition to the article by IOP Publishing of a header, an article ID, a cover sheet and/or an 'Accepted Manuscript' watermark, but excluding any other editing, typesetting or other changes made by IOP Publishing and/or its licensors"

This Accepted Manuscript is @ 2018 IOP Publishing Ltd.

During the embargo period (the 12 month period from the publication of the Version of Record of this article), the Accepted Manuscript is fully protected by copyright and cannot be reused or reposted elsewhere.

As the Version of Record of this article is going to be / has been published on a subscription basis, this Accepted Manuscript is available for reuse under a CC BY-NC-ND 3.0 licence after the 12 month embargo period.

After the embargo period, everyone is permitted to use copy and redistribute this article for non-commercial purposes only, provided that they adhere to all the terms of the licence https://creativecommons.org/licences/by-nc-nd/3.0

Although reasonable endeavours have been taken to obtain all necessary permissions from third parties to include their copyrighted content within this article, their full citation and copyright line may not be present in this Accepted Manuscript version. Before using any content from this article, please refer to the Version of Record on IOPscience once published for full citation and copyright details, as permissions will likely be required. All third party content is fully copyright protected, unless specifically stated otherwise in the figure caption in the Version of Record.

View the article online for updates and enhancements. 


\title{
Breakdown of the coherence effects and Fermi liquid behavior in $\mathrm{YbAl}_{3}$ nanoparticles
}

C. Echevarria-Bonet ${ }^{1,3}$, D P Rojas ${ }^{2}$, J I Espeso ${ }^{3}$, J Rodríguez Fernández $^{3}$, L Rodríguez Fernández ${ }^{4}$, E Bauer ${ }^{5}$, S Burdin ${ }^{6}$, S G Magalhães ${ }^{7}$, L. Fernández Barquín ${ }^{3}$

${ }^{1}$ BCMaterials, Building No. 500, Tech. Park Biscay, 48160 Derio, Spain.

${ }^{2}$ Dpto de Estructuras y Física de Edificación. ETSAM-Universidad Politécnica de Madrid, 28040 Madrid, Spain

${ }^{3}$ Dpto. CITIMAC, Facultad de Ciencias, Universidad de Cantabria, 39005 Santander, Spain ${ }^{4}$ SERMET, Universidad de Cantabria, 39005 Santander, Spain

${ }^{5}$ Institute of Solid State Physics, TU Wien, 1040 Wien, Austria

${ }^{6}$ Univ. Bordeaux, LOMA, UMR 5798, F-33400 Talence, France and CNRS, LOMA, UMR 5798, F-33400 Talence, France

${ }^{7}$ Instituto de Física, Universidade Federal do Rio Grande do Sul, 91501-970 Porto

Alegre, Brazil

E-mail: d.rojas@upm.es

\begin{abstract}
A change in the Kondo lattice behavior of bulk $\mathrm{YbAl}_{3}$ has been observed when the alloy is shaped into nanoparticles $(\approx 12 \mathrm{~nm})$. Measurements of the electrical resistivity show inhibited coherence effects and deviation from the standard Fermi liquid behavior ( $T^{2}$-dependence). The results are interpreted as due to the effect of the disruption of the periodicity of the array of Kondo ions provoked by the size reduction process. Additionally, the ensemble of randomly placed nanoparticles also triggers an extra source of electronic scattering at very low temperatures $(\approx 15 \mathrm{~K})$ due to quantum interference effects.
\end{abstract}

Keywords: Yb nanometric alloys, Kondo lattice, single impurity, electrical resistivity, Quantum interference effects

\section{Submitted to: J. Phys.: Condens. Matter}

\section{Introduction}

The behavior of magnetic impurities in a metallic host embraced in the well-known Kondo effect has been one of the most challenging problems in condensed matter physics [1]. This phenomenon is a fine example of development of electronic correlations in a solid, and in the last years it has witnessed a resurgence with the development of nanoelectronic devices and novel nanostructured materials [2, 3]. However, there is probably no other area where the presence of the Kondo effect is so fundamental as in 


\section{Breakdown of the coherence effects and Fermi liquid behavior in $Y_{b A l_{3}}$ nanoparticles 2}

Strongly Correlated Electron Systems (SCES) (Ce-, U-, and Yb-based compounds) [4]. Within the existing theories, the Kondo lattice model [5] has been successfully applied for explaining the influence of Kondo effect on the physical properties of this kind of materials.

In the framework of this model, a large number of SCES can be classified as Kondo lattice systems, and these are viewed as a dense periodic array of magnetic ions, different to the single impurity systems, where the impurities are considered far away with no coherence between each other. The possibility that long range Fermi liquid coherence may break down in a Kondo alloy has open new perspectives since it may lead to the formation of non-trivial new quantum states $[6,7,8]$. Until now, this issue has mainly been experimentally explored for bulk materials by diluting the active Kondo ion with a non magnetic one (in Ce alloys), paying an especial attention to the crossover from Kondo lattice to single impurity regime detected by electrical resistivity measurements $[9,10,11,12,13]$. Less attention has been focused on Yb alloys respect to Ce systems, with only a few reports (see for instance [14, 15, 16]), where even this crossover hardly has been explored [17].

It is also remarkable to note that most of the reports in SCES have been constrained to macroscopically well-crystallized bulk materials. However it appears evident that the development of molecular beam epitaxy techniques have allowed very recently the production of two dimensional structures based on Ce-Kondo lattice systems, known as "Kondo superlattices" [18]. The reduction of the dimensionality results in new types of electronic states, as observed in $\mathrm{CeIn}_{3}-\mathrm{LaIn}_{3}$ superlattices, where an enhancement of the effective electron mass and non Fermi liquid behavior were underlined [19]. Another example is the fabrication of multi-scale microstructures spanning from atomic to nanometer and mesoscopic scales in $\mathrm{YbAl}_{3}$ alloy, showing a simultaneous enhancement of the electron and phonon transport properties [20].

Following this alternative route, novel phenomena appear when reducing the size of those materials to the nanometer scale. In particular, it is appealing to investigate deeper the eventual variations in the relation to the role of Kondo interaction, especially in Kondo lattice compounds [21]. There are only a few examples of experimental results on Ce alloys ([22, 23] and Refs. therein), and also some theoretical approaches (see for instance $[24,25,26])$. In $\mathrm{Yb}$ systems, a significant example is the nanosized $\mathrm{YbAl}_{3}$ particles, which exhibits a decrease of the $\mathrm{Yb}$ valence with the size reduction. Such a decrease was attributed to the bonding loss of the surface atoms [27]. More recently, changes in the valence with the size reduction in $\mathrm{REPb}_{3}(\mathrm{RE}=\mathrm{Eu}, \mathrm{Yb})$ alloys were also observed [28]. For nanosized $\mathrm{YbAl}_{3}$, the bulk counterpart of this alloy is an archetypal intermediate valence system, which has been thoroughly studied in the past. The existence of energy scales related to coherence and Kondo effects allows to classify this alloy as a Kondo lattice system [14]. Electrical resistivity results indicate a decrease of the scattering around $100 \mathrm{~K}$ [29]. There is also a broad maximum around $100 \mathrm{~K}$ in the magnetic susceptibility and also in the magnetic contribution to the specific heat related to an energy scale (Kondo temperature $\left(\mathrm{T}_{K}=500-670 \mathrm{~K}\right)$ ) $[14,30,31]$, as found as well 


\section{Breakdown of the coherence effects and Fermi liquid behavior in $Y_{b A l_{3}}$ nanoparticles 3}

in the nanosized $\mathrm{YbAl}_{3}$ alloys [27]. In this kind of material with a Kondo temperature higher than $500 \mathrm{~K}$, the crystalline field effects are found to play a secondary role $[1,14,30,32]$. Moreover, studies on $\mathrm{Yb}_{1-x} \mathrm{Lu}_{x} \mathrm{Al}_{3}$ series of alloys showed that according to magnetic susceptibility and specific heat measurements the appearance of a second energy scale at $40 \mathrm{~K}$ in the intermediate valence Kondo lattice $\mathrm{YbAl}_{3}$ is a true coherence effect [14]. In addition, a reduction of Kondo lattice effects in $\mathrm{Yb}_{1-x} \mathrm{Lu}_{x} \mathrm{Al}_{3}$ was observed by soft x-ray photoelectron spectroscopy with a behavior in a good agreement with the single impurity Anderson model [15]. However, it is interesting to note that details of these changes in the Kondo lattice behavior has not been explored so far by electrical resistivity $(\rho(T))$ measurements in this Yb-substituted series $\mathrm{Yb}_{1-x} \mathrm{Lu}_{x} \mathrm{Al}_{3}$, despite it represents a smoking gun evidence to disclose the evolution of the coherence effects and the Kondo regime in strongly correlated $\mathrm{Yb}$ and Cecompounds. The Fermi liquid regime $\left(\propto T^{2}\right)$ at low temperatures and coherence effects, hallmarks of a Kondo lattice behavior, can be easily identified by measuring electronic transport properties.

Considering the electronic properties of nanosized $\mathrm{YbAl}_{3}[27]$ and those commented for the bulk alloy above, it is relevant to scrutinize the influence of the size reduction on the electronic transport properties (electrical resistivity), and how the Kondo lattice behavior and the Fermi liquid ground state ( $T^{2}$ dependence at low temperatures) are affected, both constitute the main aims of this work. This will represent a definite step forward within SCES systems (Ce, Yb,U alloys). Additionally, the results are compared with that obtained for the non-magnetic reference $\mathrm{LuAl}_{3}$ nanometric alloy, and with the dilution effect by nonmagnetic $\mathrm{Lu}$ in bulk $\mathrm{YbAl}_{3}$ alloy using the electrical resistivity as well.

\section{Experimental}

Nanocrystalline $\mathrm{LuAl}_{3}$ alloy was prepared by high-energy mechanical milling of a starting bulk alloy. Then, the alloy was crushed and milled in a planetary high-energy ball system (Retsch PM 400/2) at a rotation speed of $200 \mathrm{rpm}$, using a container and balls made of tungsten carbide, a similar procedure, as already used in the study of the $\mathrm{YbAl}_{3}$ and other Rare Earth nanoalloys [27, 33]. The bulk $\mathrm{Yb}_{0.2} \mathrm{Lu}_{0.8} \mathrm{Al}_{3}$ alloy was prepared in an arc furnace from constituent elements Yb (Alfa-4N), Lu (Alfa-4N) and Al (Alfa-5N). Transmission Electron Microscopy (TEM) was performed in a JEOL JEM 2100 microscope. X-ray diffraction measurements were performed at room temperature with a Bruker D8 Advance diffractometer using $\mathrm{Cu}-K \alpha$ radiation. Electronic transport properties were measured by the four probe method in a Quantum Design PPMS device. For the electrical resistivity measurements the milled powder is first pressed in a press pellet die. A disk is then obtained, which has to be cut in a parallelepiped shape. The sample bar was of a typical length $(10 \mathrm{~mm})$ and area $\left(2 \times 2.5 \mathrm{~mm}^{2}\right)$. Our in-house holder consisted of 4 spring-loaded pins. The pin holder was screwed on top of the sample until a certain deformation was observed in the springs. The spring-loaded pins are rounded to secure an excellent contact. 


\section{Breakdown of the coherence effects and Fermi liquid behavior in $\mathrm{YbAl}_{3}$ nanoparticles 4}

\section{Results}

Figure 1 shows the X-ray diffraction patterns and results of Rietveld refinement of 70 $\mathrm{h}$ milled $\mathrm{YbAl}_{3}$ (a) and $\mathrm{LuAl}_{3}$ (b) alloys. The Rietveld analysis of x-ray data for 70 $\mathrm{h}$ milled $\mathrm{YbAl}_{3}$ alloy (Bragg factor: $R_{B}=10 \%$ ) provided the unit-cell parameter $a=$ $4.2037(7) \AA$, mean grain size $D_{X R D}=12(2) \mathrm{nm}$ and lattice strain $\eta=0.37(3) \%$, in agreement with the previously reported results [27]. For the sample $\mathrm{LuAl}_{3}$ milled for 70 h, with a Bragg factor $\left(R_{B}=7 \%\right)$, values of $a=4.1918(4) \AA, D_{X R D}=10(2) \mathrm{nm}$ and $\eta=$ $0.64(3) \%$ are calculated. The structural characterization was completed collecting TEM images. Figure 1 (c) and (d) show representative high resolution transmission electron microscopy (HRTEM) images of the $70 \mathrm{~h}$ milled $\mathrm{YbAl}_{3}$ and $\mathrm{LuAl}_{3}$ nanoparticles. The figures show a single $\mathrm{YbAl}_{3}$ particle with a mean diameter $14 \mathrm{~nm}$ /diameter (Figure 1 (c)) and of $11 \mathrm{~nm}$ for $\mathrm{LuAl}_{3}$ nanoparticle (Figure 1(d)). The lattice planes can be clearly observed, which indicates that the nanoparticles are crystalline in the core. These values of the mean size of the nanoparticles are near to those obtained by the analysis of the $\mathrm{X}$-ray diffraction data.

Figure 2(a) shows the temperature dependence of the total electrical resistivity $(\rho(T))$ in bulk, $20 \mathrm{~h}$, and $70 \mathrm{~h}$ milled $\mathrm{YbAl}_{3}$ alloys. It is observed a gradual change in the high temperature slope in the milled samples respect to the bulk alloy, with a decrease of the relative variation in all temperature range with the milling process. For $70 \mathrm{~h}$ milled $\mathrm{YbAl}_{3}$ alloy there are two important features that indicate a remarkable difference with the bulk sample: the high temperature slope, and a minimum around $15 \mathrm{~K}$, as indicated by the marker, in more detail in figure 2(b). There is also a broad hump in the $70 \mathrm{~h}$ milled $\mathrm{YbAl}_{3}$ alloy.

The appearance of this hump is typical in the magnetic contribution to $\rho(T)$ of intermediate valence Kondo lattice systems, as observed for instance in the intermediate valence $\mathrm{YbCuAl}$ [34]. When coming down from high temperatures in $70 \mathrm{~h}$ milled $\mathrm{YbAl}_{3}$, the $\rho(T)$ increase can be attributed to the incoherent Kondo scattering (high temperature scale- $T_{K}=670 \mathrm{~K}$ [14]) until the decrease below $T_{\max }=125 \mathrm{~K}$. Thus, the contribution to the electrical resistivity in $70 \mathrm{~h}$ milled $\mathrm{YbAl}_{3}$ (see Figure 2(b)) basically comes from the magnetic scattering and the residual resistivity terms. This suggests that the disorder provoked by the milling process induces an increase of the scattering from defects in the crystal lattice, starting to play a more significant role that the electronphonon scattering. This fact has been observed in other milled Rare Earth-based alloys, where the electrical resistivity has shown a significant reduction of the high temperature slope with the increase of the milling time, with values of $\mathrm{RRR}=\rho_{300 K} / \rho_{4 K}$ (Residual Resistivity Ratio) changing from 1.6 to 1.1 [35].

On the other hand, at low temperatures a drastic change (although relatively reduced) in the behavior is observed in such a milled sample respect to the bulk alloy, with a marked upturn and the presence of a minimum around $15 \mathrm{~K}$, as shown in Figure 2(a,b). Precisely there is a small upturn in $\mathrm{YbAl}_{3} 20 \mathrm{~h}\left(\left\langle D_{X R D}\right\rangle \approx 19 \mathrm{~nm}\right.$ [27]) which demonstrates that the appearance of the minimum at $70 \mathrm{~h}$ alloy is not casual but the 


\section{Breakdown of the coherence effects and Fermi liquid behavior in $\mathrm{YbAl}_{3}$ nanoparticles $\$ 5$}

result of a definite tendency (see Figure $2(\mathrm{c})$ ). The consequence of this minimum is a deviation from standard Fermi liquid behavior, as shown in Figure 3, when comparing the bulk and $70 \mathrm{~h}$ milled $\mathrm{YbAl}_{3}$ alloy samples.

To analyze the origin of such a minimum in $\rho(T)$ it is pertinent to check $\rho(T)$ in $\mathrm{LuAl}_{3}$ milled for $70 \mathrm{~h}$. This will allow to test whether the minimum is observed in a conventional metallic alloy of a similar nanometric structure. In this case (see Figure 4 (a)), the shape of the resistivity curve is that of a typical metal. Curiously it also displays a minimum, much shallower than that in $70 \mathrm{~h}$ milled $\mathrm{YbAl}_{3}$, as shown in details in the inset of Figure 4 (a). This last feature could be intuitively interpreted as additional contributions around the minimum in the Yb-based alloy, which we will commenting below.

The analysis of the different contributions to $\rho(T)$ in disordered (amorphous) metals and nanocrystalline alloys is not a simple task. There exist several mechanisms that could provide a reasonable interpretation for the increase of the electrical resistivity at low temperatures for the nanometric $70 \mathrm{~h}$ milled $\mathrm{YbAl}_{3}$ and $\mathrm{LuAl}_{3}$ alloys. To facilitate the interpretation we will describe our rationale step by step. Firstly, it could be expected the low temperature upturn be due by eventual changes in the density of carriers, which are considered commonly in semiconducting materials and hopping conductivity. In a second step we will discuss quantum interference effects (QIE) usually observed in compounds of amorphous nature [36, 37]. Finally, a third plausible approach to quantify is the role of an eventual Kondo effect in the $\mathrm{Yb}$ sample will be discussed as another extra scattering source frequently present in SCES Yb alloys.

Regarding the first mechanism, our $\rho(T)$ measurements indicated an ohmic dependence (see Figure 4(b)), thus providing a reasonable evidence for a metallic conductivity. Thus, ruling out the influence of oxides such as $\mathrm{Yb}_{2} \mathrm{O}_{3}$ in $\rho(T)$. The presence of this oxide (less than $3 \%$ ) of the sample) has been previously detected in bulk and milled $\mathrm{YbAl}_{3}$ alloys [27]. Consequently, this ohmic dependence of $\rho(T)$ also eliminates the possibility of hopping conductivity, based on Mott metal-insulator transition, with an exponential dependence anyway [38]. Secondly, an electrical resistivity minimum has been been widely observed in amorphous materials of high $\rho_{0}(\gtrsim 80 \mu \Omega \mathrm{cm})$. The high-energy milling process usually leads to sizeable lattice strains and deformations, thus giving rise to a poorly crystallized environment at a very local range [39]. Therefore, it would not be surprising to detect an influence of these effects in $\rho(T)$. Quantum electron interaction effects [36] put forward two corrections, namely the electron-electron interaction (EEI) and the weak localization (WL). In the following, we will briefly comment on both effects. Although EEI and WL introduce temperature dependent corrections to resistivity, in three-dimensional disordered metals at low temperatures, the contribution to resistivity from the EEI effects dominates over that due to WL effects $[36,40]$. Assuming the existence of EEI, the correction to conductivity $(\sigma)$ is derived as:

$$
\Delta \sigma_{e e}=\frac{1.3}{\sqrt{2}} \frac{e^{2}}{2 \pi h} F_{0} \sqrt{\frac{2 \pi k_{B} T}{h D^{\prime}}}
$$




\section{Breakdown of the coherence effects and Fermi liquid behavior in $Y_{b A l_{3}}$ nanoparticles 6}

where $D^{\prime}=\frac{v_{F}^{2} \tau}{3}$ is the diffusion constant, in terms of the Fermi velocity $v_{F}$ and the relaxation time $\tau$. $F_{0}$ is a screening term which turns to $4 / 3$ in the case of the absence of an applied magnetic field. Experimental reports revealing the $\rho \propto-\sqrt{T}$ dependence have been extensively published for disordered metals [37]. On the other hand, the electron wave propagation, away from a classical interpretation, allows a variety of paths with the existence of loops of interference between the electron waves giving rise to a factual localization. If all particles returned back to the origin, then $\rho \rightarrow \infty$ and the full localization would be achieved.

An ideal approach to determine the influence of EEI effects is then to include $\rho \propto-\sqrt{T}$. As shown in Figure 5 (a), one can realize that it is possible to fit a linear variation ( $\sqrt{T}$ dependence) for $70 \mathrm{~h}$ milled $\mathrm{LuAl}_{3}$ alloy. This supports experimentally the former assumption: the mechanism related to the electrical resistivity minimum in $\mathrm{LuAl}_{3}$ is the commented quantum corrections.

Regarding the $\mathrm{YbAl}_{3}$ nano (70 h milled alloy), the contribution below the minimum shows an extra contribution. Let us first consider that for $T<T_{\min }$ there is a major contribution from QIE effects as occurs in $\mathrm{LuAl}_{3}$. The fit only considering the $-\sqrt{T}$ term is not satisfactory (see Figure $5(\mathrm{~b})$ ), thus suggesting the presence of another more important scattering mechanism(s). In SCES Yb systems we presented above there could exist a contribution stemming from the Kondo effect $(\rho \propto-\ln (T))[1]$. In this sense, we have found that the low temperature data $(T<7 \mathrm{~K})$, is well described according to the - $\ln \mathrm{T}$ dependence, and the results are displayed in Figure 5 (c). This could indicate the presence of an additional temperature scale $\left(T^{*}=7 \mathrm{~K}\right)$ related to the onset of an eventual Kondo contribution at low temperatures, different to the high $T_{K}$ temperature scale.

\section{Discussion}

The outcome of the former analysis is that the experimental evidence is explained by both QIE and Kondo interaction, which are responsible for the low temperature upturn below $T_{\text {min }}$. The milling of the bulk $\mathrm{YbAl}_{3}$ results in an ensemble of nanoparticles randomly dispersed and in contact, forming a granular alloy. It is commonly accepted that nanoparticles can be generally described as a two-component system consisting of the core nanocrystallites and a grain or interphase boundary components [41]. The thickness of this grain boundary is commonly assumed around $1 \mathrm{~nm}$. At grain boundaries, more disorder is expected in relation to the core of nanoparticles. On the one hand, the nanometric size of the particles favors the presence of surface shells in which the atomic coordination is reduced whereas the microstrain is enhanced. In this situation the nanostructure becomes more and more disordered, affecting the electrical resistivity. Therefore, such a considerable disorder is the basic element supporting the QIE.

In bulk $\mathrm{YbAl}_{3}$, the $f$-electron atoms are forming a periodic array which lie in the socalled Kondo lattice systems [5]. Every spin in the lattice is screened by the conduction 


\section{Breakdown of the coherence effects and Fermi liquid behavior in $Y_{b A l_{3}}$ nanoparticles 7}

electrons. In real space, the screening takes place on a length scale of mesoscopic size ( $\xi_{K}=\hbar v_{F} / k_{B} T_{K}$, where $v_{F}$ is the Fermi velocity), denoted as the Kondo screening cloud [42]. The values of Fermi velocity may range from $10^{6} \mathrm{~m} / \mathrm{s}$ for normal metals to $10^{3}-10^{4}$ $\mathrm{m} / \mathrm{s}$ in Heavy Fermion systems [43]. Consequently, the Fermi velocity is directly related with the electronic contribution to the specific heat (Sommerfeld coefficient $(\gamma)$ ). The value of $\gamma=45 \mathrm{~mJ} / \mathrm{molK}^{2}$ estimated from the specific heat data in bulk $\mathrm{YbAl}_{3}$ is found to decrease down to $36 \mathrm{~mJ} / \mathrm{molK}^{2}$ for the $70 \mathrm{~h}$ milled $\mathrm{YbAl}_{3}$ alloy [27]. Since a value of the Fermi velocity $v_{F}=4 \cdot 10^{4} \mathrm{~m} / \mathrm{s}$, is observed for the bulk $\mathrm{YbAl}_{3}$ [44], a simple scaling with the variation of the coefficient $\gamma$ leads to an appraisal of $5 \cdot 10^{4} \mathrm{~m} / \mathrm{s}$ for the Fermi velocity in the milled sample. On the other hand, from the maximum in the magnetic susceptibility and magnetic contribution to the specific heat in bulk and milled $\mathrm{YbAl}_{3}$ alloys [27], the $T_{K}$ values do not change significantly, being around $500 \mathrm{~K}-670$ $\mathrm{K}$, as estimated for the bulk sample $[14,30,32]$. Thus, following the above reasoning, it is possible to estimate a value of $\xi_{K}$ around $0.7 \mathrm{~nm}$ for the $70 \mathrm{~h}$ milled $\mathrm{YbAl}_{3}$ alloy. The length scale $\xi_{K}$ would be less than the mean size of our samples (12 nm), and consequently the Kondo screening cloud is restricted within the nanoparticle, at least in the core, with variations at the surface of the nanoparticles. The size effect would not be essential for the disruption of the Kondo lattice behavior, as the characteristic length is one order of magnitude lower than the mean grain size, whereas the increase in the lattice strain and the disorder at the surface of the particles with the size reduction process will play a significant role. Our measurements of electrical resistivity of nanosized $\mathrm{YbAl}_{3}$ indicate that the nanoparticle ensemble provokes the breakdown of the Kondo lattice coherence, with a presence of a low temperature upturn.

It has been shown that the reduction of size in nano- $\mathrm{YbAl}_{3}$ results in the $\mathrm{Yb}$ valence reduction as a consequence of the increasing non-magnetic $\mathrm{Yb}^{2+}$ atoms at the surface of nanoparticles [27]. Irrespective of this qualitative interpretation, what is clear is that by reducing the size, a $\rho(T)$ minimum appears with the low temperature upturn. As a matter of fact, this situation resembles the cases of bulk alloys under chemical substitution in which by adding non-magnetic rare-earth atoms ( $\mathrm{Y}$ and La for Ce-based and La or Lu for Yb-based compounds) the transition from Kondo Lattice to single impurity regime is observed (see for instance $[12,17]$. In bulk $\mathrm{YbAl}_{3}$ the coherence scattering is maintained throughout the alloy as a result of a Kondo lattice behavior. This is greatly modified whenever there is a reduction of size and a concomitant increase of surface strain and disorder in the nanoparticle shell. For comparison purposes, the possibility of a change of regime by Lu dilution was explored in the bulk $\mathrm{YbAl}_{3}$. The bulk sample $\mathrm{Yb}_{0.2} \mathrm{Lu}_{0.8} \mathrm{Al}_{3}$ is found to crystallize in the same type of structure and space group $P m-3 m$ as $\mathrm{YbAl}_{3}$ and $\mathrm{LuAl}_{3}$ alloys but with a lattice parameter $a=$ $4.1932(2) \AA$. The results show the presence of a low temperature minimum in $\rho(T)$ of the bulk $\mathrm{Yb}_{0.2} \mathrm{Lu}_{0.8} \mathrm{Al}_{3}$ alloy with an upturn following a $-\ln \mathrm{T}$ dependence (see Figure 6 ). Curiously, the position of this minimum around $7 \mathrm{~K}$, coincides with the onset of $-\ln T$ term below the temperature scale $T^{*}$, associated to the low temperature upturn in the $70 \mathrm{~h}$ milled $\mathrm{YbAl}_{3}$ alloy. Consequently, it is possible to find some similarities 


\section{Breakdown of the coherence effects and Fermi liquid behavior in $\mathrm{YbAl}_{3}$ nanoparticles 8}

between the size reduction and chemical substitution process by $\mathrm{Lu}$ in bulk $\mathrm{YbAl}_{3}$ alloy. In both of cases there is an increase in the fraction of non-magnetic atoms $\left(\mathrm{Yb}^{2+}\right.$ or $\mathrm{Lu}$ ) and the disorder leading to a limit situation where the periodicity of the-Kondo ions is broken. Consequently, a deviation from the Fermi liquid regime is observed.

Concerning the observed - $\ln \mathrm{T}$ dependence of the low temperature upturn in the $70 \mathrm{~h}$ milled $\mathrm{YbAl}_{3}$ alloy, it is obvious that the electronic transport in an ensemble of nanoparticles is complex. It will require further study in order to ascribe it to a Kondo behavior.

\section{Conclusions}

It is concluded that the size in intimate combination with the increasing strain is a magnificent driving parameter, now for intrinsic electronic scattering changes. In this sense and to extend the universality of the former assert, it is widely known that particles of noble metals in nanocrystalline state can suffer serious changes in the density of states, as have been recently reported [45]. Therefore, our finding is naturally connected with a more general physical situation.

To summarize, the scrutiny of the electrical resistivity in nano- $\mathrm{YbAl}_{3}$ particles has revealed a deviation from the Fermi liquid behavior at low temperatures. Such a deviation is due to the combined effect of the disruption of the periodicity of the Kondo ions as consequence of size reduction process and QIE favored by the presence of a disordered collection of nanoparticles.

\section{Acknowledgements}

This work was supported by the Spanish MINECO under project MAT2014-55049-C2R.

[1] Hewson A C 1997 The Kondo problem to Heavy fermions (Cambridge, England: Cambridge University Press)

[2] Goldhaber-Gordon D, Shtrikman H, Mahalu D, Abusch-Magder D, Meirav U and Kastner M A 1998 Nature 391156

[3] Prüser H, Dargel P E, Bouhassoune M, Ulbrich R G, Pruschke T, Lounis S and Wenderoth M 2014 Nat. Commun. 55417

[4] Coleman P 2007 Handbook of Magnetism and Advanced Magnetic Materials (Chichester: Wiley)

[5] Doniach S 1977 Physica B 91231

[6] Burdin S and Fulde P 2007 Phys. Rev. B $\mathbf{7 6} 104425$

[7] Kaul R K and Vojta M 2007 Phys. Rev. B 75132407

[8] Burdin S and Lacroix C 2013 Phys. Rev. Lett. 110226403

[9] Lawrence J M, Graf T, Hundley M F, Mandrus D, Thompson J D, Lacerda A, Torikachvili M S, Sarrao J L and Fisk Z 1996 Phys. Rev. B 5312559

[10] Bud'ko S L, Fontes M B and Baggio-Saitovitch E M 1998 J. Phys.: Condens. Matter 108815

[11] Chudinov S, Brando M, Marcelli A and Battisti M 1998 Physica B 244154

[12] Medina A N, Rojas D P, Gandra F G, Azanha W R and Cardoso L P 1999 Phys. Rev. B 59(13) 8738

[13] Pikul A P, Stockert U, Steppke A, Cichorek T, Hartmann S, Caroca-Canales N, Oeschler N, Brando M, Geibel C and Steglich F 2012 Phys. Rev. Lett. 108066405 
Breakdown of the coherence effects and Fermi liquid behavior in $\mathrm{YbAl}_{3}$ nanoparticles 9

[14] Bauer E D, Booth C H, Laurence J M, Hundley M F, Sarrao J L, Thompson J D, Riseborough P S and Ebihara T 2004 Phys. Rev. B 69125102

[15] Yamaguchi J, Sekiyama A, Imada S, Yamasaki A, Tsunekawa M, Muro T, Ebihara T, Onuki Y and Suga S 2007 New J. Phys. 9317

[16] Köhler U, Oeschler N, Steglich F, Maquilon S and Fisk Z 2008 Phys. Rev. B 77(10) 104412

[17] Rojas D P, Medina A N, Gandra F G, Fernández Barquín L and Gómez Sal J C in press Physica $B$

[18] Shimozawa M, Goh S K, Shibauchi T and Matsuda Y 2016 Reports on Progress in Physics 79 074503

[19] Shishido H, Shibauchi T, Yasu K, Kato T, Kontani H, Terashima T and Matsuda Y 2010 Science $\mathbf{3 2 7} 980$

[20] He D, Zhao W, Mu X, Zhou H, Wei P, Zhu W, Nie X, Su X, Liu H, He J and Zhang Q 2017 Journal of Alloys and Compounds 7251297 - 1303

[21] Lonzarich G, Pines D and feng Yang Y 2017 Reports on Progress in Physics 80024501

[22] Chen Y Y, Huang P H, Ou M N, Wang C R, Yao Y D, Lee T K, Yao M Y, Laurence J M and Booth C H 2007 Phys. Rev. Lett. 98157206

[23] Mohanta S K, Mishra S N, Iyer K K and Sampathkumaran E V 2013/ Phys. Rev. B 87(12) 125125

[24] Thimm W B, Kroha J and von Delft J 1999 Phys. Rev. Lett. 822143

[25] Hand T, Kroha J and Monien H 2006 Phys. Rev. Lett. 97136604

[26] Schwabe A, Gütersloh D and Potthoff M 2012 Phys. Rev. Lett. 109257202

[27] Rojas D P, Barquín L F, Espeso J I and Rodríguez Fernández J 2008 Phys. Rev. B 78094412

[28] Subbarao U, Sarkar S, Jana R, Bera S S and Peter S C 2016 Inorganic Chemistry 55 5603-5611

[29] Rowe D M, Kuznetsov V L, Kuznetsova L A and Min G'2002 J. Phys. D: Appl. Phys. 352183

[30] Cornelius A L, Laurence J M, Ebihara T, Riseborough P S, Booth C H, Hundley M F, Pagliuso P G, Sarrao J L, Thompson J D, Jung M H, Lacerda A H and Kwei G H 2002 Phys. Rev. Lett. 88117201

[31] Burdin S, Georges A and Grempel D R 2000 Phys. Rev. Lett. 851048

[32] Ebihara T, Bauer E D, Cornelius A L, Laurence J M, Harrison N, Thompson J D, Sarrao J L, Hundley M F and Uji S 2003 Phys. Rev, Lett. 90166404

[33] Rojas D P, Barquín F, Fernández J R, Espeso J I and Sal J C G 2007 Journal of Physics: Condensed Matter 19186214

[34] Schlottmann P 1989 Physics Reports 1811

[35] Echevarria-Bonet C, Rojas D P, Espeso J I, Fernández J R, de la Fuente Rodríguez M, Barquín L F, Fernández L R, Gorria P, Blanco J A, Fdez-Gubieda M L, Bauer E and Damay F 2015 Journal of Physics: Condensed Matter 27496002

[36] Lee P A and Ramakrishnan T V 1985 Rev. Mod. Phys. 57287

[37] Howson M A and Gallagher B L 1988 Phys. Reports 170265

[38] Kittel C 1986 Introduction to Solid State Physics (New York: John Wiley \& Sons)

[39] Echevarria-Bonet C, Rojas D P, Espeso J I, Rodríguez Fernández J, Rodríguez Fernández L, Gorria P, Blanco J A, Fdez-Gubieda M L, Bauer E, André G and Fernández Barquín L 2013 Phys. Rev. B 87(18) 180407

[40] Ghosh T, Fukuda T, Kakeshita T, Kaul S N and Mukhopadhyay P K 2017 Phys. Rev. B 95(14) 140401

[41] Rojas D P, Barquín L F, Fernández J R, Fernández L R and Gonzalez J 2010 Nanotechnology 21 445702

[42] Park J, Lee S S B, Oreg Y and Sim H S 2013 Phys. Rev. Lett. 110(24) 246603

[43] Mourachkine A 2004 Room-temperature Superconductivity (Cambridge, UK: Cambridge International Science Pub.)

[44] Demsar J, Kabanov V V, Alexandrov A S, Lee H J, Bauer E D, Sarrao J L and Taylor A J 2009 Phys. Rev. B 80(8) 085121

[45] Garitaonandia J S, Insausti M, Goikolea E, Suzuki M, Cashion J D, Kawamura N, Ohsawa H, 
Breakdown of the coherence effects and Fermi liquid behavior in $\mathrm{YbAl}_{3}$ nanoparticles10

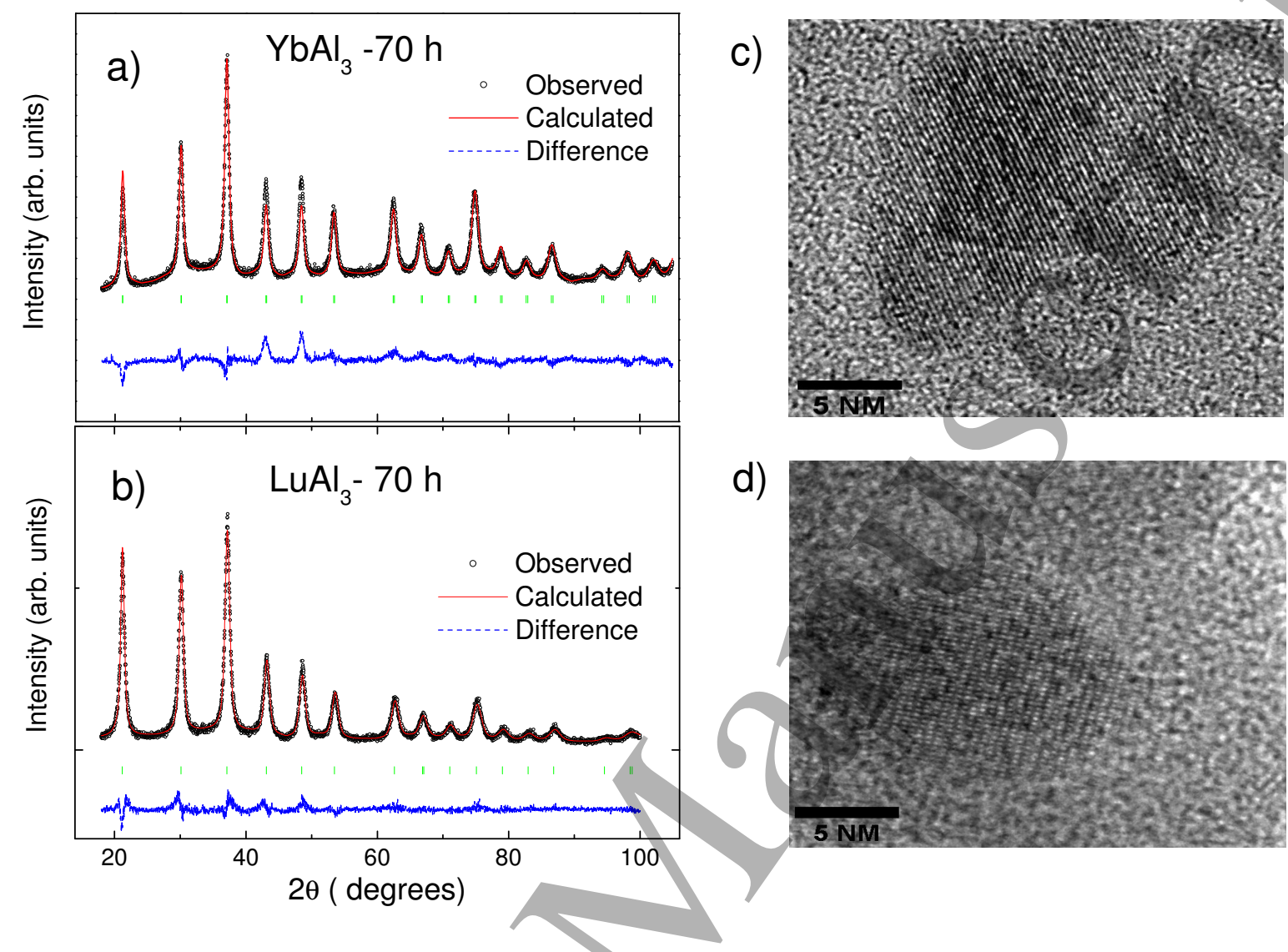

Figure 1. X-ray diffraction patterns and results of Rietveld refinement of $70 \mathrm{~h}$ milled $\mathrm{YbAl}_{3}$ (a) and $\mathrm{LuAl}_{3}$ (b) alloys. The vertical markers are the expected Bragg positions according to $P m$-3m space group. High resolution TEM image of a nanoparticle of 70 $\mathrm{h}$ milled $\mathrm{YbAl}_{3}$ (c) and $\mathrm{LuAl}_{3}(\mathrm{~d})$ alloys.

Gil de Muro I, Suzuki K, Plazaola F and Rojo T 2008 Nano Lett. 8661 

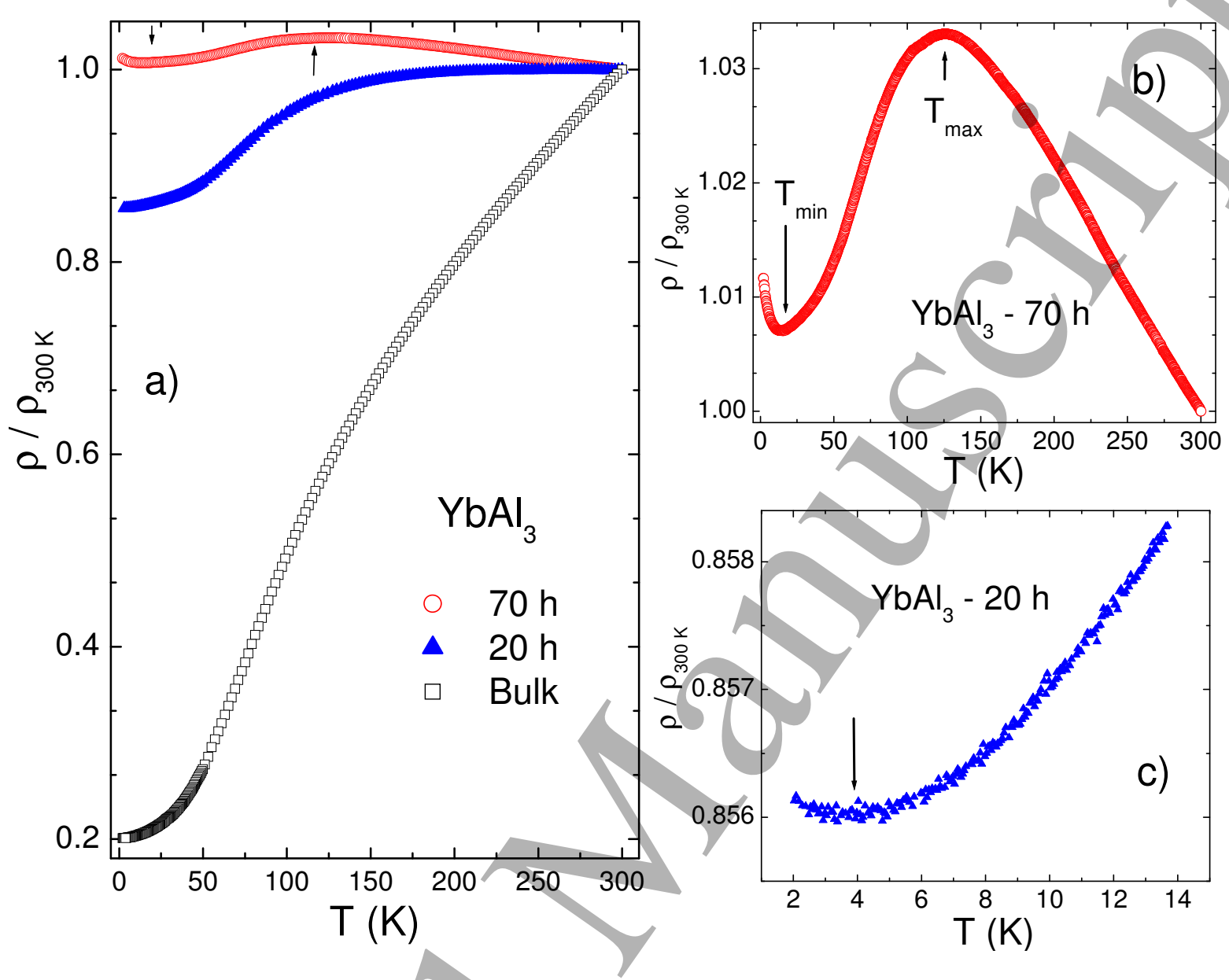

Figure 2. a) Normalized electrical resistivity vs temperature for bulk, $20 \mathrm{~h}$ and $70 \mathrm{~h}$ milled $\mathrm{YbAl}_{3}$ alloys. In the milled sample, there is a minimum followed by an increase of the resistivity, up to a broad maximum, as indicated by the markers. b) Details of normalized electrical resistivity curve for 70 milled $\mathrm{YbAl}_{3}$ alloy, highlighting the existence of a temperature minimum and maximum. c) Details of the low temperature region of $20 \mathrm{~h} \mathrm{YbAl}$ milled alloy showing a minimum in $\rho(T)$ around $4 \mathrm{~K}$. 
Breakdown of the coherence effects and Fermi liquid behavior in $\mathrm{YbAl}_{3}$ nanoparticles 12

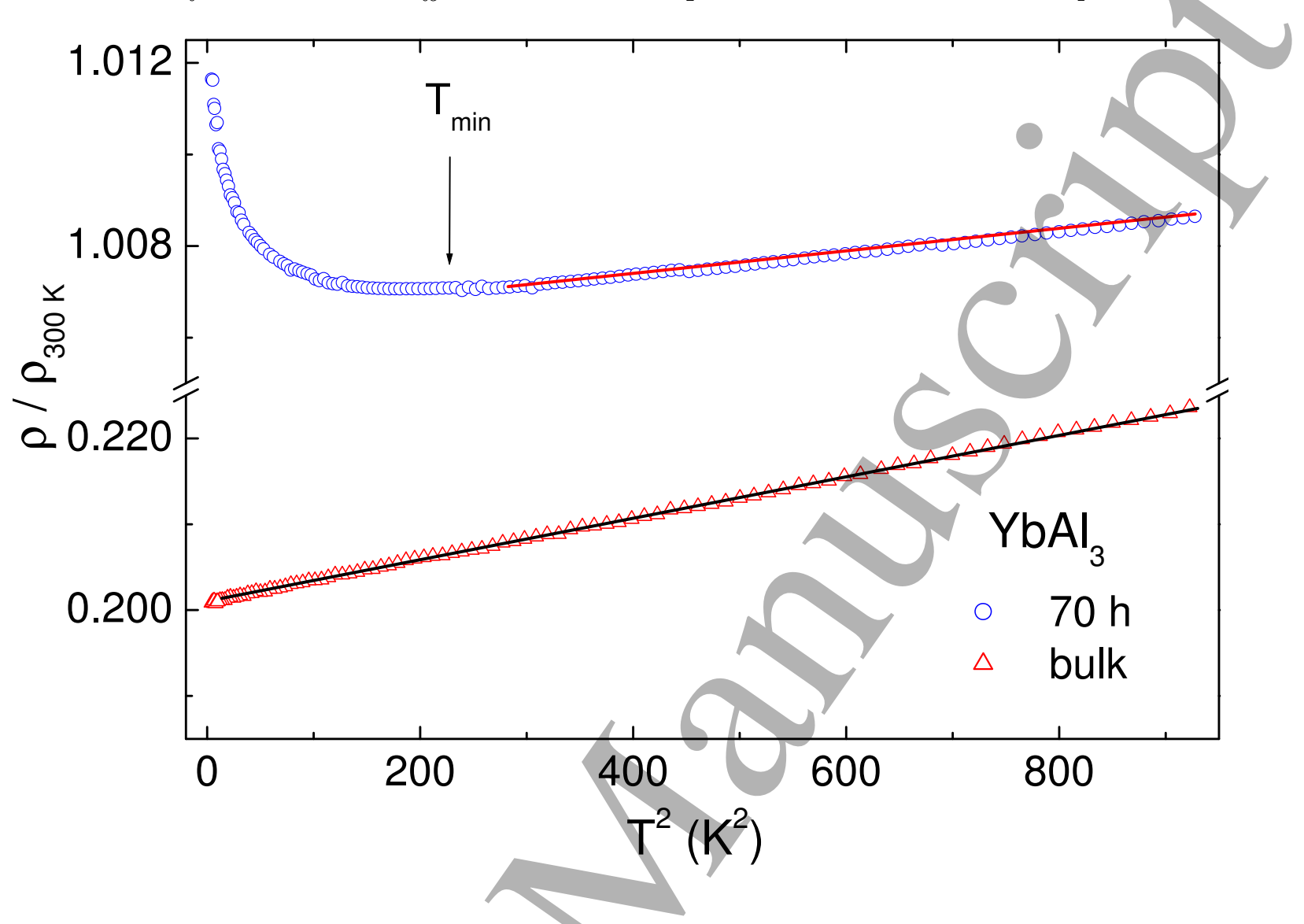

Figure 3. Details of the low temperature region of bulk and $70 \mathrm{~h}$ milled $\mathrm{YbAl}_{3}$ alloys showing a deviation from the Fermi liquid dependence below $\mathrm{T}_{\min }$ in the nanometric alloy. 
Breakdown of the coherence effects and Fermi liquid behavior in $\mathrm{YbAl}_{3}$ nanoparticles 13

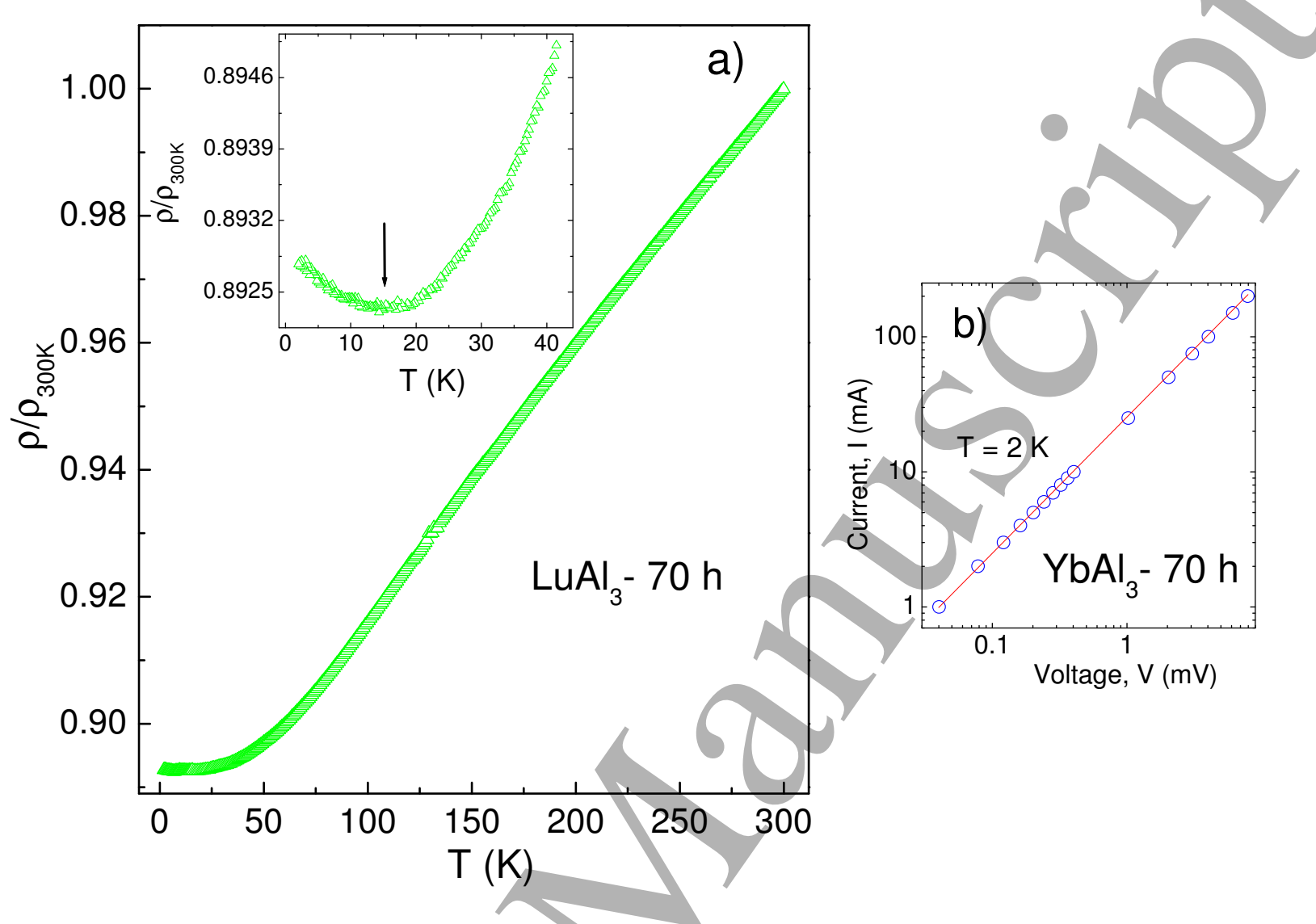

Figure 4. a) Normalized electrical resistivity curve of the 70 milled $\mathrm{LuAl}_{3}$ alloy. The inset details the low temperature region showing the presence of a minimum around $15 \mathrm{~K} . \mathrm{b})$ Curve of applied current (I) vs. voltage (V) in log-log scale at $2 \mathrm{~K}$ in the 70 $\mathrm{h}$ milled $\mathrm{YbAl}_{3}$ alloy. 
Breakdown of the coherence effects and Fermi liquid behavior in $\mathrm{YbAl}_{3}$ nanoparticles 14

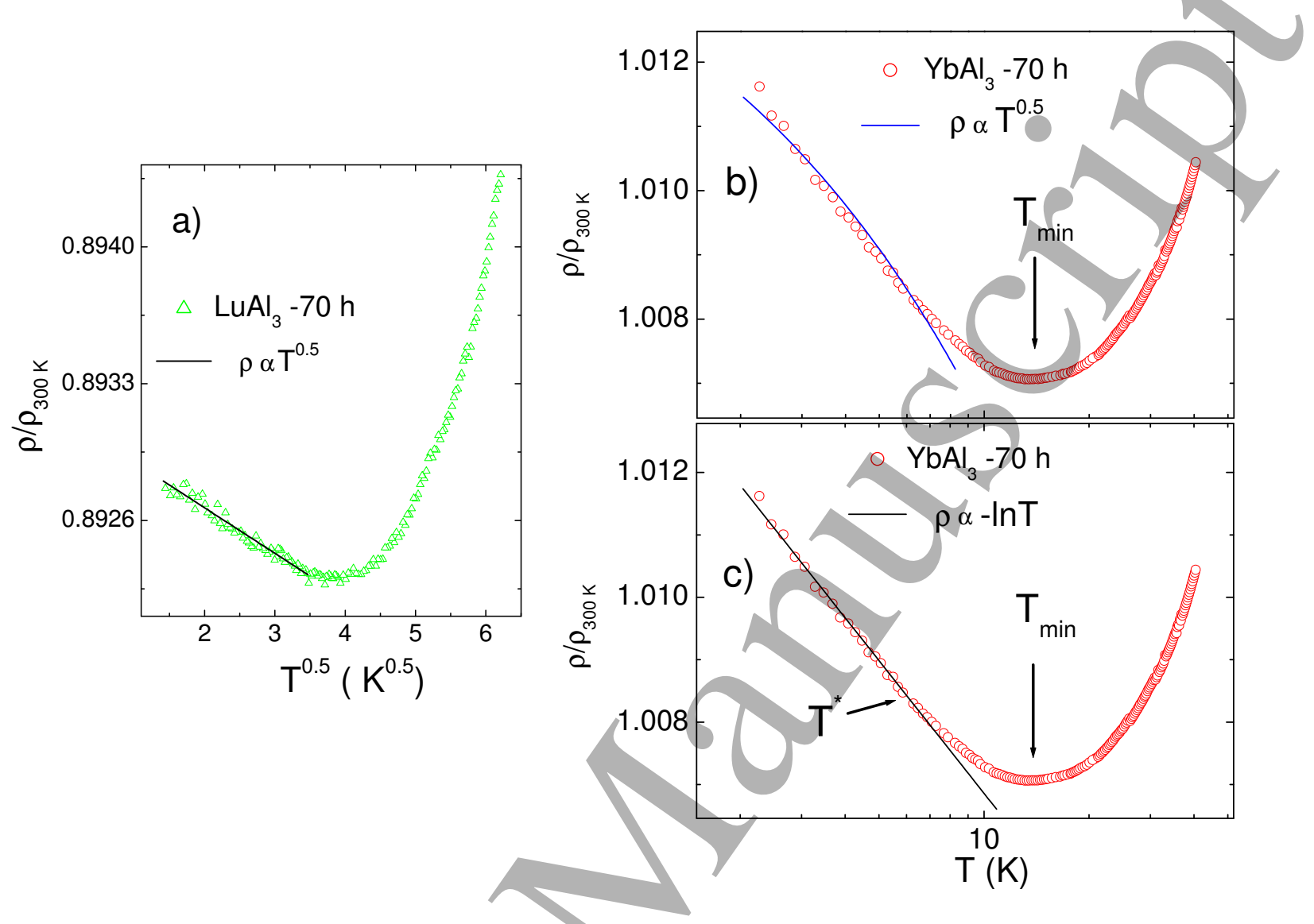

Figure 5. a) Low temperature region of electrical resistivity described by a $-\sqrt{T}$ term in $70 \mathrm{~h}$ milled $\mathrm{LuAl}_{3}$. Fitting of the electrical resistivity data in $70 \mathrm{~h}$ milled $\mathrm{YbAl}_{3}$ alloy in logarithmic scale, according to $-\sqrt{T}$ term (b) and a $-\ln T$ dependence (c). In the $\mathrm{Yb}$ alloy, the data is better described by a $-\ln T$ behavior below $T^{*}=7 \mathrm{~K}$. 
Breakdown of the coherence effects and Fermi liquid behavior in $\mathrm{YbAl}_{3}$ nanoparticles 15

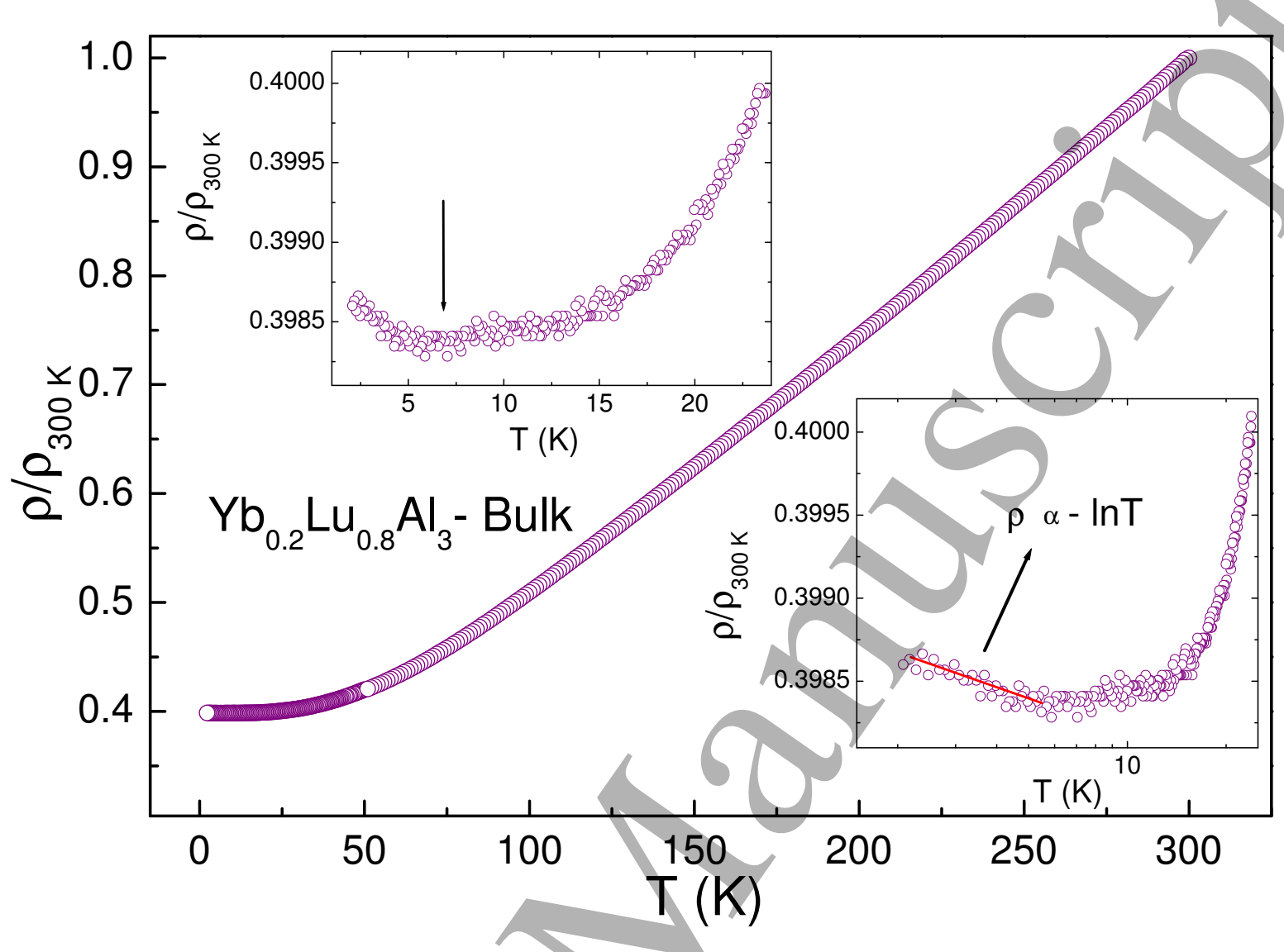

Figure 6. Normalized electrical resistivity curve of $\mathrm{Yb}_{0.2} \mathrm{Lu}_{0.8} \mathrm{Al}_{3}$ bulk alloy. Details of the low temperature region showing the presence of a minimum around $7 \mathrm{~K}$ (left inset), with an upturn according to -lnT dependence (right inset). 